\title{
SOME REMARKS ON MAGNETIC DIPOLE MOMENTS IN LIGHT NUCLEI
}

\author{
J.F.A. Van HIENEN and P.W.M. GLAUDEMANS \\ Fysisch Laboratorium der Rijksuniversiteit, Utrecht, The Netherlands
}

Received 26 October 1972

\begin{abstract}
Multi-shell mixed-configuration calculations employing various effective interactions show that for low-lying states the $g$-factor in $T_{z}=0$ nuclei as well as the average of $g$-factors of corresponding states in mirror nuclei varies between +0.4 and $+0.9 \mathrm{n} . \mathrm{m}$. in rather good agreement with a pure $j^{n}$ description; for excited states in even-even $T_{z}=0$ nuclei one finds $g \approx+0.50 \mathrm{n} . \mathrm{m}$. The results agree well with present experimental data on magnetic dipole moments of ground states and excited states.
\end{abstract}

A comparison of experimentally observed multipole moments with theoretical values may provide a direct test of the corresponding wave functions, since in contrast with most other observables, the theoretical description of only one specific state is involved.

The magnetic dipole moment of a state $|J\rangle$ is given by

$\mu=\left\langle J M\left|\sum_{k=1}^{A}\left(g_{k}^{l} l_{k}+g_{k}^{s} s_{k}\right)\right| J M\right\rangle_{M=J}$,

where $g^{d}$ and $g^{s}$ denote the nucleon orbital and spin $g$-factors, respectively. Writing the matrix element in isospin formalism as a sum of an isoscalar term $\mu_{0}$ and an isovector term $\mu_{z}$, yields

$\mu=\mu_{\mathrm{o}}+T_{z} \mu_{z}$,

where the $T_{z}$ dependence of the isovector contribution is shown explicitly. This discussion will be restricted to the isoscalar part $\mu_{0}$, which can be obtained directly from experimentally determined magnetic dipole moments in $T_{z}=0$ nuclei and by averaging the dipole moments of corresponding states in mirror nuclei with the relation

$\mu_{\mathrm{o}}=1 / 2\left[\mu\left(A, T_{z}\right)+\mu\left(A,-T_{z}\right)\right]$.

It can be shown $[1,2]$ that the measured isoscalar contribution to the magnetic dipole moment for nuclei described by a closed major shell and one participle or hole is very well reproduced by the Schmidt single-particle estimate. An analysis of the intrinsic spin contribution to the isoscalar part has been given by Leonardi and Rosa-Clot [3]. These authors show that the observed mass dependence of the intrinsic spin contribution can be explained by taking into ac- count some configuration mixing.

It might be useful, however, to point out the simple behaviour of the isoscalar term as a function of mass number. In this paper the isoscalar contribution as obtained from multi-shell mixed-configuration wave functions is described. Substitution of bare-nucleon spin and orbital $g$-factors in eq. (1) leads to

$\mu=g_{0} J=1 / 2 J+0.38\left\langle\sum_{k=1}^{A} s_{k}\right\rangle$,

where the numerical factor is given by $1 / 2\left(g_{\mathrm{p}}^{s}+g_{\mathrm{n}}^{s}-1\right)$ [3]. For a pure $j^{n}$ configuration one can derive from expressions given in ref. [4] and the proton and neutron single-particle Schmidt values with $g_{0}=1 / 2\left(g_{p}+g_{n}\right)$ the relation

$g_{0}=1 / 2 \pm \frac{0.38}{2 l+1}$ for $j=l \pm 1 / 2$.

The values of $g_{0}$ derived from eq. (5) for nuclei with $A \leqslant 41$ are compared with experiment in fig. 1 . More details about the experimental data are given in table 1. Values of $g_{0}$ calculated from the multi-shell mixedconfiguration wave functions of refs. [5-12] are shown also. It follows that the isoscalar part varies only slightly with mass number within the $1 \mathrm{~s}_{1 / 2}$, $1 p_{3 / 2}$ and $1 p_{1 / 2}$ subshells in agreement with the lin. ear mass dependence assumed in ref. [3]. This linear mass dependence is not found, however, for the $1 d_{5 / 2}, 2 s_{1 / 2}$ and $1 d_{3 / 2}$ subshells. It can be seen from fig. 1 that the $g_{0}$-values obtained from eq. (5) agree well with experiment as well as with the $g_{0}$-values obtained from the much more complicated shell model wave functions.

The deviations at $A=6$ and 19 (points (a) and (b) 


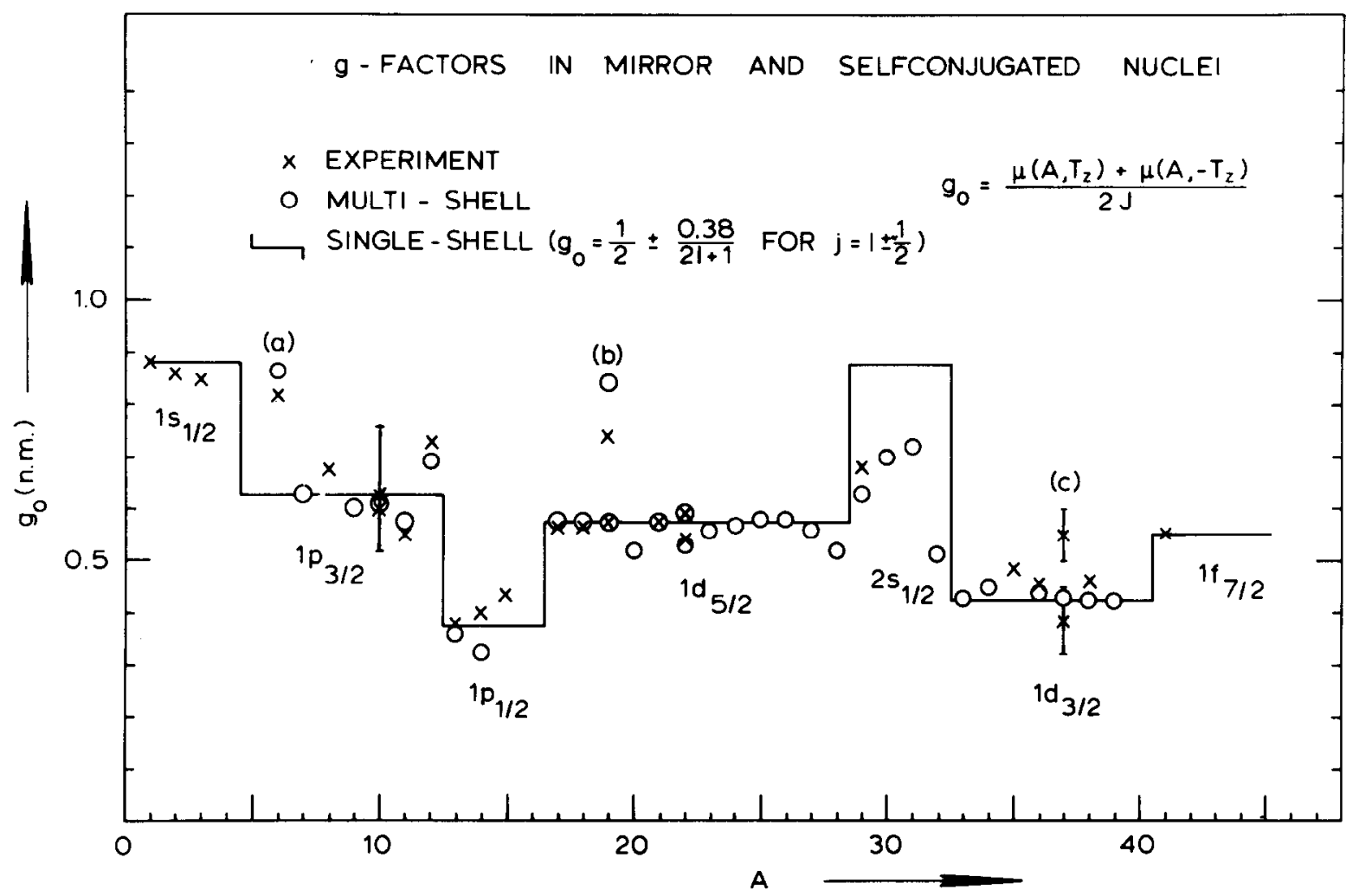

Fig. 1. Comparison between theory and experiment for $g_{0}$-factors in mirror and self-conjugated nuclei. The theoretical multi-shell values are obtained from refs. [5-12]. The points marked (a), (b) and (c) are discussed in the text.

in fig. 1) are explained by configurations that contain strong $\mathrm{p}_{3 / 2} \mathrm{p}_{1 / 2}$ and $\mathrm{s}_{1 / 2}$ contribu tions, respectively. The multi-shell wave functions seem to overestimate these components, however. The value at $A=37$ (marked with (c)) refers to a $J^{\pi}=7 / 2-$ excited state and should, as it does, correspond with the $f_{7 / 2}$ single-shell estimate. A deviation occurs also near mass 30 . The simplest description of these nuclei in terms of a pure $\left(\mathrm{s}_{1 / 2}\right)^{n}$ configuration gives a $g_{0}$-value which is too large. The values calculated with more complex wave functions follow from strong admix tures of $d_{5 / 2}$ and $d_{3 / 2}$ configurations.

The fact that the calculated $g_{0}$-value of a given state is rather independent of the theoretical wave function can be illustrated with calculations on the lowest $J^{\pi}=2^{+}$state in ${ }^{24} \mathrm{Mg}$. The $g_{0}$-value obtained with eq. (5) is +0.576 n.m., while mixed configuration wave functions [8] employing empirical as well as surfacedelta matrix elements both yield $g_{0}=0.565$ n.m. A projected Hartree-Fock calculation [13] gives also the value $g_{0}=+0.565$ n.m. A similar behaviour has been found in other $T_{z}=0$ nuclei. This shows that the last term in eq. (4) is generally found to be much smaller than $0.5 \mathrm{n} . \mathrm{m}$. and has a fixed sign in a given subshell. From a study of the theoretically calculated isoscalar contributions in light nuclei $(A<40)$ as well as in $\mathrm{Ni}$ isotopes [14], one finds for low-lying states in eveneven nuclei the relation

$\left.\ll \sum_{k=1}^{A} s_{k}\right\rangle \mid<0.4$

With eqs. (6) and (4) one can estimate the magnetic dipole moment of e.g. the lowest $2^{+}$state in eveneven $T_{z}=0$ nuclei, which leads to the value $\mu=+1.00 \pm 0.16$ n.m..

It may thus be concluded that the experimentally determined isoscalar contribution to the magnetic dipole moment of light nuclei can be reproduced rather 
Table 1

Experimental values of the isoscalar $g_{0}$-factors $^{a}$ ) for mirror and selfconjugated nuclei $b$ )

\begin{tabular}{|c|c|c|c|c|c|c|c|c|c|c|c|}
\hline & $J^{\pi}$ & $T$ & $g_{\mathrm{o}}^{\exp }$ & & $J^{\pi}$ & $T$ & $g_{0}^{\exp }$ & & $J^{\pi}$ & $T$ & $g_{0}^{\exp }$ \\
\hline $\mathrm{n} / \mathrm{H}$ & $1 / 2^{+}$ & $1 / 2$ & +0.88 & ${ }^{13} \mathrm{C} / \mathrm{N}$ & $1 / 2^{-}$ & $1 / 2$ & +0.38 & ${ }^{22} \mathrm{Na}$ & $1^{+}$ & 0 & +0.54 \\
\hline${ }^{2} \mathrm{H}$ & $1^{+}$ & 0 & +0.86 & ${ }^{14} \mathrm{~N}$ & $1^{+}$ & 0 & +0.40 & ${ }^{29} \mathrm{Si} / \mathrm{P}$ & $1 / 2^{+}$ & $1 / 2$ & +0.68 \\
\hline${ }^{3} \mathrm{H} / \mathrm{He}$ & $1 / 2^{+}$ & $1 / 2$ & +0.85 & ${ }^{15} \mathrm{~N} / \mathrm{O}$ & $1 / 2^{-}$ & $1 / 2$ & +0.44 & ${ }^{35} \mathrm{Cl} / \mathrm{Ar}$ & $3 / 2^{+}$ & $1 / 2$ & +0.48 \\
\hline${ }^{6} \mathrm{Li}$ & $1^{+}$ & 0 & +0.82 & ${ }^{17} \mathrm{O} / \mathrm{F}$ & $5 / 2^{+}$ & $1 / 2$ & +0.57 & ${ }^{36} \mathrm{Cl} / \mathrm{K}$ & $2^{+}$ & 1 & +0.46 \\
\hline${ }^{8} \mathrm{Li} / \mathrm{B}$ & $2^{+}$ & 1 & +0.57 & ${ }^{18} \mathrm{~F}$ & $5^{+}$ & 0 & +0.57 & ${ }^{37} \mathrm{Ar} / \mathrm{K}$ & $3 / 2^{+}$ & $1 / 2$ & $+0.38 \pm 0.07$ \\
\hline${ }^{10} \mathrm{~B}$ & $3^{+}$ & 0 & +0.60 & ${ }^{19} \mathrm{~F} / \mathrm{Ne}$ & $1 / 2^{+}$ & $1 / 2$ & +0.74 & ${ }^{37} \mathrm{Ar} / \mathrm{K}$ & $7 / 2^{-}$ & $1 / 2$ & $+0.55 \pm 0.04$ \\
\hline${ }^{10} \mathrm{~B}$ & $1^{+}$ & 0 & $+0.63 \pm 0.12$ & ${ }^{19} \mathrm{~F} / \mathrm{Ne}$ & $5 / 2^{+}$ & $1 / 2$ & +0.57 & ${ }^{38} \mathrm{~K}$ & $3^{+}$ & 0 & +0.46 \\
\hline${ }^{11} \mathrm{~B} / \mathrm{C}$ & $3 / 2^{-}$ & $1 / 2$ & +0.55 & ${ }^{21} \mathrm{Ne} / \mathrm{Na}$ & $3 / 2^{+}$ & $1 / 2$ & +0.57 & ${ }^{41} \mathrm{Ca} / \mathrm{Sc}$ & $7 / 2^{-}$ & $1 / 2$ & +0.55 \\
\hline${ }^{12} \mathrm{~B} / \mathrm{N}$ & $1^{+}$ & 1 & +0.73 & ${ }^{22} \mathrm{Na}$ & $3^{+}$ & 0 & +0.58 & & & & \\
\hline
\end{tabular}

a) See eqs. (3) and (4).

b) Errors smaller than the last digit are not given.

accurately with a simple $j^{n}$ configuration. Much more complex multi-shell mixed-configuration wave functions do not produce considerably different results. The $g$-factors obtained for a $j^{n}$ configuration may therefore be useful to obtain an estimate of the $g$-factor in $T_{z}=0$ nuclei and also for the $g$-factor of a state of which the value of the corresponding state in the mirror nucleus is known experimentally. Finally, it should be remarked that the calculated isovector term contributing to the magnetic dipole moment of $T_{z} \neq 0$ nuclei depends rather strongly in the size of the configuration space in contrast to the isoscalar term.

This work was performed as part of the research program of the "Stichting voor Fundamenteel Onderzoek der Materie" (F.O.M.) with financial support from the "Nederlandse Organisatie voor Zuiver Wetenschappelijk Onderzoek" (Z.W.O.).

\section{References}

[1] M. Ichumira and K. Yazaki, Nucl. Phys. 63 (1965) 401.

[2] H.A. Mavromatis and L. Zamick, Phys. Lett. 20 (1966) 171.

[3] R. Leonardi and M. Rosa-Clot, Phys. Rev. Lett. 24 (1970) 407 .

[4] A. de Shalit and I. Talmi, Nuclear shell theory (Acad. Press, New York and London, 1963).

[5] S. Varma and P. Goldhammer, Nucl. Phys. A125 (1969) 193.

[6] E.C. Halbert, J.B. McGrory, B.H. Wildenthal, S.P. Pandya, Advances in nuclear physics 4 (1971) 315 .

[7] B.M. Preedom and B.H. Wildenthal, Michigan State University, private communication .

[8] J.F.A. Van Hienen, to be published.

[9] M.J.A. de Voigt, P.W.M. Glaudemans, J. De Boer and B.H. Wildenthal, Nucl. Phys. A186 (1972) 365.

[10] P.W.M Glaudemans, P.M. Endt and A.E.L. Dieperink, Ann. of Phys. 63 (1971) 134.

[11] B.H. Wildenthal, J.B. McGrory, E.C. Halbert and H.D. Graber, Phys. Rev. C4 (1971) 1708.

[12] B.H. Wildenthal, E.C. Halbert, J.B. McGrory and T.T.S. Kuo, Phys. Rev. C4 (1971) 1266.

[13] M.R. Gunye, Phys. Lett. 37B (1971) 125.

[14] P.W.M. Glaudemans, M.J.A. De Voigt and E.F.M. Steffens, Nucl. Phys. to be published. 